\title{
Effects of early life permethrin exposure on spatial working memory and on monoamine levels in different brain areas of pre-senescent rats
}

\author{
Cinzia Nasuti, Manuel Carloni, Donatella Fedeli, Rosita Gabbianelli, Antonio Di Stefano, Cerasa \\ Laura Serafina, Isabel Silva, Valentina Domingues, Roberto Ciccocioppo
}

\begin{abstract}
A B S T R A C T
Pesticide exposure during brain development could represent an important risk factor for the onset of neurodegenerative diseases. Previous studies investigated the effect of permethrin (PERM) administered at $34 \mathrm{mg} / \mathrm{kg}$, a dose close to the no observable adverse effect level (NOAEL) from post natal day (PND) 6 to PND 21 in rats. Despite the PERM dose did not elicited overt signs of toxicity (i.e. normal body weight gain curve), it was able to induce striatal neurodegeneration (dopamine and Nurr1 reduction, and lipid peroxidation increase). The present study was designed to characterize the cognitive deficits in the current animal model. When during late adulthood PERM treated rats were tested for spatial working memory performances in a T-maze-rewarded alternation task they took longer to choose for the correct arm in comparison to age matched controls. No differences between groups were found in anxiety-like state, locomotor activity, feeding behavior and spatial orientation task. Our findings showing a selective effect of PERM treatment on the T-maze task point to an involvement of frontal cortico-striatal circuitry rather than to a role for the hippocampus. The predominant disturbances concern the dopamine (DA) depletion in the striatum and, the serotonin (5-HT) and noradrenaline (NE) unbalance together with a hypometabolic state in the medial prefrontal cortex area. In the hippocampus, an increase of $\mathrm{NE}$ and a decrease of DA were observed in PERM treated rats as compared to controls. The concentration of the most representative marker for pyrethroid exposure (3-phenoxybenzoic acid) measured in the urine of rodents $12 \mathrm{~h}$ after the last treatment was $41.50 \mu \mathrm{g} / \mathrm{L}$ and it was completely eliminated after $96 \mathrm{~h}$.
\end{abstract}

Keywords:

Rat

Permethrin

Early life exposure

3-Phenoxybenzoic acid

Spatial working memory

T-maze

Monoamines

\section{Introduction}

Pyrethroids are among the most frequently used pesticides worldwide. They are used in agriculture, public health, and homes, as well as for the protection of textiles, such as carpets. Because these insecticides are relatively nonvolatile, the primary source of exposure is believed to be through diet. Additional exposure via ingestion of contaminated household dust may occur after the indoor application of pesticides such as permethrin that is the main pyrethroid used in the home for indoor pest control, in pet shampoos and treatment for wood furniture.

Population-based biomonitoring data for pyrethroid metabolites measured in US and German population revealed that pyrethroid insecticide exposure is widespread. The presence of its metabolites in the urine of U.S. and German residents indicates that children may have higher exposures than adolescents and adults (Barr et al., 2010; Heudorf and Angerer, 2001). A developing brain is much more susceptible to the toxic effects of chemicals than an adult brain. This vulnerability period extends from fetal development through infancy, childhood and adolescence. The biological effects of pyrethroid are in part caused by their ability to alter neuronal activity since they interact with specific binding sites of voltage-gated sodium channel (VGSC) slowing the rate of VGSC closing, prolonging the inward sodium conductance and then shifting the membrane to more polarized potentials (Narahashi, 1996). A secondary consequence to cell membrane depolarization is an increased $\mathrm{Ca}^{+2}$ influx into the neurons through voltage-gated calcium channel (VGCC) that contributes to impact neuronal synaptic plasticity of neurons (Imamura et al., 2006). These changes in synaptic transmission may alter neuronal function and may contribute to excitotoxicity and neurodegenerative pathology (Gomez-Villafuertes et al., 2007).

Studies conducted in our laboratory demonstrated that PERM exposure, at a dose of $34 \mathrm{mg} / \mathrm{kg}$ close to the NOAEL $(25 \mathrm{mg} / \mathrm{kg})$ during early life, induced, later in the life of rats, a significant neurodegeneration of the striatum (Str) characterized by a decrease of 
Nurr1 gene and protein expression, and an increased lipid peroxidation (Carloni et al., 2012). Lower DA levels and accelerated DA turnover following early life PERM treatment was also observed (Nasuti et al., 2007). Nurr1 is a transcription factor belonging to the nuclear receptor family and it regulates the development and maintenance of dopaminergic neurons. In fact, our previous studies demonstrated that early life permethrin treatment impaired the Nurr1 expression and then the dopaminergic system.

To further explore the impact of early life PERM exposure, in the present study, we sought to investigate cognitive functions related to spatial working memory in pre-senescent rats that were exposed to this insecticide during the neonatal period. Concomitantly with spatial working memory tasks, DA, NE, 5-HT and their major metabolites levels from different brain areas were also measured ex vivo.

\section{Materials and methods}

\subsection{Materials}

All reagents were of pure analytical grade. Technical grade (75:25, trans:cis; 94\% purity) 3-phenoxybenzyl-(1R,S)-cis,trans-3-(2,2-dichlorovinyl)-2,2dimethylcyclopropanecarboxyl-ate, PERM (NRDC 143) were generously donated by Dr. A. Stefanini of ACTIVA (Milan, Italy). Corn oil, dopamine, 3,4-dihydroxy phenylacetic acid (DOPAC), 3-methoxy-4-hydroxyphenylacetic acid (HVA), dihydroxybenzylamine (DHBA), 1-octanesulphonic acid sodium salt, sodium metabisulphite and acetonitrile were obtained from Sigma (Milan, Italy).

\subsection{Animals}

Male and female Wistar rats from Charles River (Calco, LC, Italy), weighing 250-270 g and about 90 days old were housed in plastic (Makrolon) cages (five rats per cage $)$ in a temperature controlled room $\left(21 \pm 5^{\circ} \mathrm{C}\right)$ and $60 \%$ humidity on $12 \mathrm{~h}$ light/dark inverted cycle (lights on at 7:00 p.m.) and maintained on a laboratory diet with water ad libitum. Rat pups born in our laboratory from primiparous dams were used in the study. The parturition day was set as PND0. On PND1, all litters were examined externally for the presence of gross abnormalities, sexed, weighed, the female pups were discarded and two male pups were assigned to each dam until weaning (PND21) for this study. No cross-fostering was employed. At 2 days of age, litters were randomly assigned to two experimental groups ( $n=11$ pups for each). Rats were weighed at 4-week intervals throughout the course of study.

At PND21, six treated and 6 control rats were housed for 4 days in metabolic cages and submitted to 3-phenoxybenzoic acid measurement in the urine.

On pre-senescent age (PND500), control and PERM treated rats ( $n=7$ per group) were subjected to a battery of behavioral tests comprising the T-maze test, water maze spatial reference memory task, open field (OF) test and elevated plus-maze. Home cage food intake of pre-senescent rats was also measured. In a subsequent experiment, 3 control and 3 PERM treated rats were trained on the T-maze task for three days. On day four, at completion of the T-maze experiment, rats were sacrificed, brains were removed and the medial prefrontal cortex (mPFC), the hippocampus (Hip) and the Str were dissected out for biochemical analysis. All procedures were conducted in adherence to the European Community Council Directive for Care and Use of Laboratory Animals.

\subsection{Treatment}

PERM was dissolved in corn oil and administered by gavage at the dose of $34.05 \mathrm{mg} / \mathrm{kg} / 4 \mathrm{ml}$, which corresponds to $1 / 50$ of $\mathrm{LD}_{50}$ as determined in adult rats (Cantalamessa, 1993). PERM or its vehicle (corn oil, $4 \mathrm{ml} / \mathrm{kg}$ ) was administered once a day from PND6 to PND21. PERM dose was adjusted daily based on rat pups body weight. On PND21, the offspring were weaned and the littermates were housed together. For the experiments, the experimental groups were formed by drawing animals from different litters, so that no group contained siblings.

\subsection{Urine 3-phenoxybenzoic acid levels}

3-Phenoxybenzoic acid (3-PBA) is the main metabolite of the PERM and other pyrethroids resulting from the oxidation of the 3-phenoxybenzyl alcohol. Its concentration in the urine is the most representative marker for pyrethroid exposure in rodents (Nakamura et al., 2007) and humans (Leng et al., 1997).

To assess PERM levels, after the last treatment day (PND 21), 6 control and 6 PERM treated rats were housed in metabolic cages for 4 days. Every $12 \mathrm{~h}$, the urine samples were collected and the volumes were measured. Urines were immediately frozen at $-20^{\circ} \mathrm{C}$ until assays were performed. Immediately prior to analytical detection, a hydrolysis step was introduced to convert potentially conjugated 3-PBA into free 3-PBA. The 2-PBA was added to urine samples and used as an internal standard. Briefly, urine samples were pre-treated with $\mathrm{KOH}$ and then, by solid-phase extrac- tion using Strata X C cartridge, the 3- and the 2-PBA were isolated. Derivatization of 3-PBA and 2-PBA was achieved by addition of DIC and HFIP. At this point, $1 \mu \mathrm{L}$ of upper layer was injected on a Thermo Trace-Ultra gas chromatograph coupled to an ion trap mass detector Thermo Polaris, operated in the electron impact ionization at $70 \mathrm{eV}$. The ion source temperature and the MS transfer temperature were at $250^{\circ} \mathrm{C}$. Operating in the splitless mode, helium was used as carrier gas at a constant flow rate of $1.3 \mathrm{~mL} / \mathrm{min}$. The injector was maintained at $240^{\circ} \mathrm{C}$. Final values of 3-PBA at $12,24,48,72$ and $96 \mathrm{~h}$ were expressed as $\mu \mathrm{g} / \mathrm{L}$ and then multiplied for the corresponding urine volume so that the quantity of 3-PBA per each urine sample was obtained.

\subsection{T-maze apparatus}

Spatial non-matching-to-place testing was conducted on an enclosed-T maze made of clear plexiglas T-shaped platform with a start $\operatorname{arm}(70 \mathrm{~cm}$ long and $11 \mathrm{~cm}$ wide) and two identical goal arms ( $51 \mathrm{~cm}$ long and $11 \mathrm{~cm}$ wide), all arms were bordered with 11-cm-high walls. At the distal end of each arm a barrier ( $3 \mathrm{~cm} \mathrm{high)}$ concealed a food well to contain the food reward ( $0.1 \mathrm{~g}$ pellet). A small grid compartment containing inaccessible fresh food was located at the end of both arms to mask the food odor (coming from the well) to prevent the use of olfactory cues to locate the food. Apparatus arms were wiped with $10 \%$ alcohol solution and dried with a paper towel between trials to eliminate the use of local odor cues. The room was surrounded by extramaze spatial cues and was illuminated by a dim light to reduce rat anxiety.

\subsection{T-maze testing}

The T-maze test is classically used for spatial working memory studies in the rat. In the $T$ maze, rats are trained to choose between two goal arms alternatively rewarded with food and the rat is trained to visit the rewarded arm to obtain it. Daily, training session is composed of several choice trials. Animals' behavior is scored for accuracy (\% correct arm choice), perseveration (number of consecutive incorrect trials) and choice reaction time (CRT) for each trial.

In the present study, one group of control $(N=7)$ and one group of PERM $(N=7)$ treated rats were used. One week prior to the beginning of the experiment, rats were housed in individual cages and mildly food deprived (85\% of their voluntary daily food intake). T-maze training started by moving the animals into the testing room where they were kept for $10 \mathrm{~min}$ prior to initiate the performance. Animals were then positioned on the maze where they had free access to food in both arms for $10 \mathrm{~min}$ a day for 2 consecutive days. Starting from day 3, rats were subjected to 11 daily consecutive choice trials. In the first daily trial, both goal arms were rewarded and the rat was asked to choose one of the two arms. In the second trial, only the arm opposite to the first choice was baited. The test continued for other 9 daily trials in which the two goal arms were rewarded with an alternated sequence. At the beginning of each trial, the rat was placed on the start arm of the T make where it was confined with a wood block for $5 \mathrm{~s}$. The block was then removed and the animal allowed to run toward the two goal arms and to choose one of them. After the choice, the rat was confined in the goal arm (using a wood block) and allowed to eat the food until finished. Rats were subjected this training for 7 consecutive days ( 10 choice trials per day) for a total of 70 trials.

The experiment was replicated in a new group of rats $(N=6)$. Specifically, 3 controls and 3 PERM treated animals were subjected to the same experimental sequence described above but the choice tests were stopped on day 4 when, immediately after completion of the behavioral task, the rats were sacrificed and the brains were removed for neurochemical analysis. Day 4 was when the maximal difference in the T maze performance between controls and PERM treated rats was observed.

\subsection{Morris Water maze testing}

One day after completion of the T-maze testing, controls $(N=7)$ and PERM $(N=7)$ treated rats were trained in the hidden-platform water maze task. The maze consisted of a large, circular tank (diameter $150 \mathrm{~cm}$; wall height $60 \mathrm{~cm}$ ) filled with water $\left(23-25^{\circ} \mathrm{C}\right)$ and placed in the center of a large room with extramaze cues. An escape platform was located $1 \mathrm{~cm}$ beneath the water surface in a fixed position and a nontoxic black paint was added to obscure the platform and to aid tracking of the animals' swim paths. In order to secure them from the water, the rats had to find a hidden escape platform located in the same position across all days of training. Animal performance was tracked by video camera using EthoVision 7.0 software (Noldus Information Technology, The Netherlands). Briefly, rats received four trials per day for five consecutive days using a $30 \mathrm{sec}$ intertrial interval. On each training trial, rats were placed in the water and allowed to swim until finding the platform or for $90 \mathrm{~s}$ at which time they were placed on the platform by the experimenter. Once on the platform, rats were allowed stay for $30 \mathrm{~s}$ to acquire memory of its position using the surrounding cues. The start position for each trial was semi-randomly varied among four equally spaced positions around the perimeter of the pool. Latency to escape platform was the dependent measure, with shorter latency time indicating better performance. Mean proximity to escape platform was recorded as the primary measure of the animals' cognitive ability based on demonstration of its superior sensitivity compared to alternative measures (Gallagher et al., 1993). Lower scores, on 
the proximity to escape platform, indicate a more accurate search because of short distance of rat to the platform during the trial.

\subsection{Locomotor activity}

One day after completion of the water maze task, controls $(N=7)$ and PERM $(N=7)$ treated rats were tested for their locomotor behavior using automated locomotor activity boxes (Med Associates, VT 05478) as previously reported (Nasuti et al., 2008). Locomotor activity was recorded for $10 \mathrm{~min}$, starting $1 \mathrm{~min}$ after placing the animal in the test cage. Each rat was automatically recorded by interruptions of two orthogonal light beams $(3.5 \mathrm{~cm}$ and $13.0 \mathrm{~cm}$ above the activity box floor), which were connected to automatic software (Activity Monitor, Med Associates). The behavioral parameters observed were ambulatory (number of horizontal episodes), rearing (number of vertical episodes), and stereotype counts (number of grooming movements). The OF was divided into two squares and, ambulatory, rearing and stereotype counts into the central area $(25 \mathrm{~cm} \times 25 \mathrm{~cm})$ which can be considered an unprotected area for rats, were also recorded. These parameters are expressed as percentage of total ambulatory, rearing and stereotype counts, respectively. Low percentage of activities in the center area indicates a higher anxiety-like state.

\subsection{Elevated plus maze}

One day after completion of the locomotor activity test, controls $(N=7)$ and $\operatorname{PERM}(N=7)$ treated rats were evaluated in the elevated plus maze (EPM) to analyze their anxiety-like behavior. After completion of the locomotor activity testing, the anxiety-like behavior of rats was assessed by a cross shaped wood made elevated maze, consisting of two opposite open arms $50 \mathrm{~cm} \times 10 \mathrm{~cm}$, and two opposite arms enclosed by $40 \mathrm{~cm}$ high walls with two open arms and two closed arms. The maze was elevated $50 \mathrm{~cm}$ from the floor and lit by dim light. A closed-circuit TV camera was mounted vertically over the maze, and the numbers of entries and time spent in enclosed and open arms were tracked by EthoVision software. Changes in the percentage of time spent and number of entries into the open arms indicate changes in anxiety-like behavior (Pellow et al., 1985). A greater number of entries and time spent in open arms means less anxiety-like condition.

\subsection{Food intake measurement}

Daily home cage food intake of control $(N=7)$ and PERM $(N=7)$ treated rats were measured during one week. Food consumption was monitored the week after the last behavioral test took place.

\subsection{Monoamines and metabolites measurements in Str, Hip and mPFC}

On day 4 of the T-maze training, at completion of the task, controls $(N=3)$ and $\operatorname{PERM}(\mathrm{N}=3)$ treated rats were killed by $\mathrm{CO}_{2}$, brains were rapidly removed, and the Hip, the Str and the mPFC were dissected as described by Spijker (2011) according to the rat brain atlas coordinates (Paxinos and Watson, 2007). Specifically, the mPFC was hand-dissected from the coronal slice $\sim 2.5-4.5 \mathrm{~mm}$ anterior to bregma and included the prelimbic cortex, part of the infralimbic and cingulate cortices. The two halves of the Str (dorsal and ventral) and of the Hip were separately hand-dissected out. The two halves of Hip was hand-dissected from the slice $\sim 3.5-5.5 \mathrm{~mm}$ posterior to bregma and included dentate gyrus and CA1-3 hippocampal regions. Tissue was stored at $-70^{\circ} \mathrm{C}$ until used for monoamine, glucose and lactate analysis.

Levels of catecholamines (DA, DOPAC, HVA, NE) were analyzed with a Waters 600 controller pump, a Rheodyne 7295 injector with a $10 \mu \mathrm{L}$ loop, and an Antec Leyden Decade II detector (the operating potential was $0.75 \mathrm{~V}$ ). Separation was achieved on a Waters Symmetry RP-C18 column $(4.6 \mathrm{~mm} \times 150 \mathrm{~mm}, 5 \mu \mathrm{m})$. The mobile phase consisted of $0.045 \mathrm{M}$ monobasic sodium phosphate, $0.001 \mathrm{M}$ 1-octanesulphonic acid sodium salt, $0.006 \%$ triethylamine, $0.015 \% 100 \mu \mathrm{M}$ sodium EDTA and $6 \%$ acetonitrile. The $\mathrm{pH}$ of the mobile phase was adjusted to 3.0 by o-phosphoric acid. The mobile phase was filtered and degassed by vacuum. A flow rate of $1 \mathrm{~mL} / \mathrm{min}$ was used in all experiments (Cannazza et al., 2005). The separation of 5-HT and its metabolite, 5-HIAA, were carried out with the flow-rate set at $1.20 \mathrm{~mL} / \mathrm{min}$ and the detector potential set at $+780 \mathrm{mV}$ vs. $\mathrm{Ag} / \mathrm{AgCl}$ reference electrode. For the mobile phase, buffer $6.90 \mathrm{~g}$ of $\mathrm{NaH}_{2} \mathrm{PO}_{4} \cdot \mathrm{H}_{2} \mathrm{O}(50 \mathrm{mM}), 37 \mathrm{mg}$ of $\mathrm{Na}_{2}$ EDTA $(0.1 \mathrm{mM})$ and $702 \mathrm{mg}$ of 1-octanesulfonic acid sodium salt monohydrate $(2.3 \mathrm{mM})$ were dissolved in UHQ water. After the $\mathrm{pH}$ was lowered to 3.0 with phosphoric acid, the final volume was adjusted to $1 \mathrm{~L}$ with UHQ water. A total of $790 \mathrm{~mL}$ of the buffer was then mixed with $210 \mathrm{~mL}$ of acetonitrile. The chromatographic separation of the main metabolite of NE, MHPG, was achieved with the flow-rate set at $1.20 \mathrm{~mL} / \mathrm{min}$ and the detector potential was $+800 \mathrm{mV}$ vs. $\mathrm{Ag} / \mathrm{AgCl}$ reference electrode. The mobile phase was a mixture of $5 \%$ of methanol and $95 \%$ of an aqueous solution of $10.5 \mathrm{~g} / \mathrm{L}$ citric acid, $20 \mathrm{mg} / \mathrm{L}$ EDTA and $20 \mathrm{mg} / \mathrm{L}$ OSA buffered at $\mathrm{pH} 3.5$ with $1 \mathrm{~mol} / \mathrm{L} \mathrm{NaOH}$. The flow rate was $1 \mathrm{~mL} / \mathrm{min}$. Each compound was identified on the basis of retention time and the concentration was established from the peak area ratio using DHBA as internal standard. Final values were expressed in terms of nanogram per gram of tissue. Measurements were performed in triplicate for each original sample.

\subsection{Glucose and lactate levels in Str, Hip and mPFC}

In the CNS, glucose is metabolized by astrocytes to produce lactate that is then released from astrocytes and utilized by neurons as energy source (Tsacapoulos and Magistretti, 1996; Pellerin, 2003). A correct balance between glucose (low level) and lactate (high level) reveals a correct energy metabolism of neurons. On the contrary, an insufficient lactate production indicates poor metabolic neuronal function and reduced protection against insult (Uehara et al., 2012; Wyss et al., 2011; Newman et al., 2011).

Hence, to monitor neuronal metabolism, glucose and lactate levels in the Str Hip and MPFC were measured.

According to the method of Bleiberg et al. (1991), a reversed-phase highperformance liquid chromatography (RP-HPLC) separation method with UV detection was developed for the detection of lactate and glucose. All analyses were carried out on a Waters 600 controller pump, a Rheodyne 7295 injector with a $10 \mu \mathrm{L}$ loop, equipped with a Waters 2996 photodiode array detector. The chromatographic analysis of lactate was carried out with a Symmetry RP-C18 column $(4.6 \mathrm{~mm} \times 150 \mathrm{~mm}, 5 \mu \mathrm{m})$; the mobile phase was a phosphoric acid solution ( $\mathrm{pH}$ 2.5 ) with $5 \%$ acetonitrile. The flow rate was $1.0 \mathrm{~mL} / \mathrm{min}$; the lactate was detected at $210 \mathrm{~nm}$. The detection of glucose was carried out on a Supelcosil $\mathrm{LC}-\mathrm{NH}_{2}$ column $(4.6 \mathrm{~mm} \times 250 \mathrm{~mm}, 5 \mu \mathrm{m})$; the mobile phase was a mixture of $75 \%$ of acetonitrile and $25 \%$ of water and UV was set at $190 \mathrm{~nm}$. The flow rate was $2.0 \mathrm{~mL} / \mathrm{min}$. Final values were expressed as nanogram per gram of tissue.

\subsection{Statistical analysis}

For the T-maze and the Morris water maze tests, a two-way ANOVA with one factor within (time) and one factor between (treatment) was employed. The EPM, the OF and the food intake tests were analyzed by mean of a one-way (treatment) ANOVA. Monoamine, glucose and lactate levels in the different brain areas were evaluated separately with nonparametric Mann-Whitney- $U$ test. When appropriate post-hoc analysis was carried out using the Newman-Keuls test. Statistical significance was set at $P<0.05$.

\section{Results}

\subsection{Effect of PERM on body weight}

Rats treated with vehicle or PERM did not show gross signs of abnormalities throughout the experimental period. During the course of the 500 days of observation, no significant group differences $(F[1,12]=4.37, P>0.05)$ in body weight were observed. A significant time effect indicated that the growth rate was similar in both groups $(F[16,192]=253, P<0.01)$.

\subsection{Urine 3-phenoxybenzoic acid levels}

Results showed that, in rats treated with PERM, $90 \%$ of the $3-P B A$ was excreted within the first $24 \mathrm{~h}$. Specifically, the amount of 3-PBA excreted at $12 \mathrm{~h}$ and $24 \mathrm{~h}$ following the last PERM administration was $0.11 \pm 0.01$ and $0.01 \pm 0.004 \mu \mathrm{g}$, respectively. The 3-PBA was completely eliminated after $96 \mathrm{~h}$ (Fig. 1).

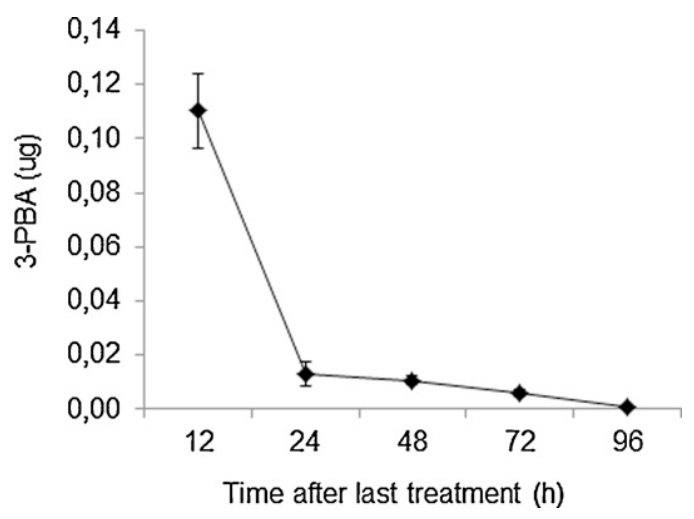

Fig. 1. 3-PBA urinary level measured $12,24,48,72$ and $96 \mathrm{~h}$ after the rats are treated per o.s.: subchronically from PND6 to PND21 with permethrin $(34 \mathrm{mg} / \mathrm{kg} / 4 \mathrm{ml})$. All data are expressed as mean \pm SEM for six rats. 

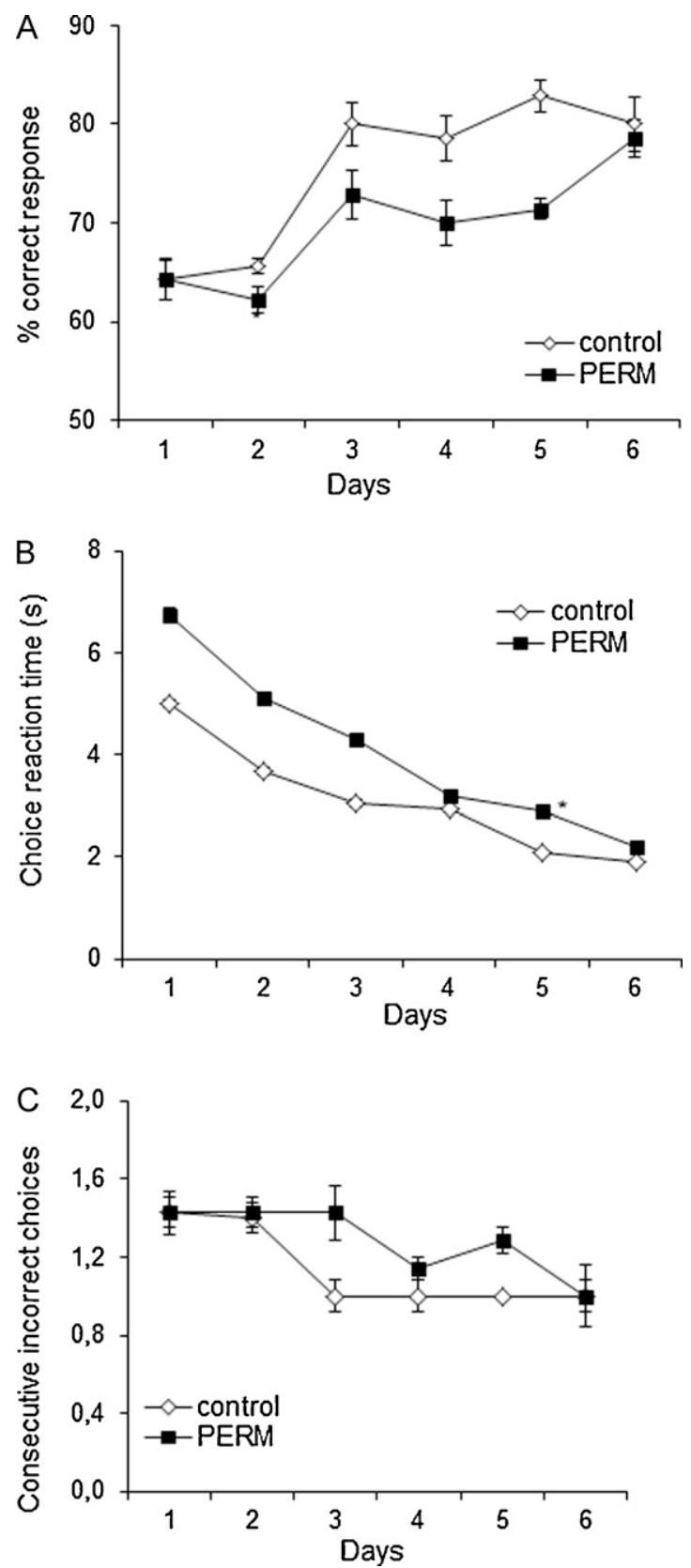

Fig. 2. Effects of early life PERM treatment on T-maze task in pre-senescent rats. (A) \% correct responses, (B) choice reaction time and (C) numbers of incorrect arm choices made in consecutive trials during six days of testing (10 trials/day). All data are expressed as mean \pm SEM. Group sizes: control $(N=7)$, PERM $(N=7)$.

\subsection{Effect of PERM on spatial working memory in the T-maze task}

ANOVA did not reveal significant main effects of PERM treatment $(F[1,12]=4.12, P=0.065)$ nor treatment $\times$ time interaction $(F[5,60]=0.32, P>0.05)$, whereas a significant effect of time $(F[5,60]=3.41, P<0.01)$ was observed (Fig. $2 \mathrm{~A})$.
On the other hand, when CRT responses were analyzed (Fig. 2B), ANOVA revealed a significant main effect of treatment $(F[1,138]=8.48, P<0.01)$ and day $(F[5,690]=12.49, P<0.001)$, but not a significant treatment $\times$ day interaction $(F[5,690]=0.59$, $P>0.05$ ). Post hoc Newman-Keuls test demonstrated that CRT was significantly longer in the PERM treated group compared with that of the controls $(P<0.01)$.

The last dependent variable analyzed was the number of incorrect arm choices made in consecutive trials (Fig. 2C). ANOVA failed to reveal a significant main effect of treatment $(F[1,12]=3.2$, $P>0.05)$ and treatment $\times$ day interaction $(F[5,60]=0.17, P>0.05)$.

\subsection{Effect of PERM on spatial reference memory in the Morris water maze}

In animals trained to search a hidden-platform in a fixed location, ANOVA revealed no significant treatment differences $(F[1,54]=1.54, P>0.05)$ and treatment $\times$ day interaction $(F[4,216]=0.3, P>0.05)$ on escape latencies. A significant time effect $(F[4,216]=70.82, P<0.01)$ indicated that the rate of task acquisition was similar in both groups. When proximity to escape platform was analyzed by ANOVA, results revealed no significant effect of treatment $(F[1,54]=0.99, P>0.05)$ and treatment $\times$ day interaction $(F[4,216]=0.3, P>0.05)$. A significant effect of time on proximity to escape platform $(F[4,216]=73.48, P<0.01)$ indicated similar rate of task acquisition in both groups.

\subsection{Effect of PERM on locomotor activities in the OF}

As shown in Table 1, in the OF test, ANOVA revealed no differences in the horizontal $(F[1,12]=0.32, P>0.05)$ and in the vertical $(F[1,12]=0.01, P>0.05)$ activities in the two groups of rats. ANOVA, also revealed absence of differences in locomotor activity (\% ambulatory counts: $F[1,12]=0.66, P>0.05 ; \%$ vertical counts: $F[1,12]=0.03$, $P>0.05$ ) occurring in the central area of the OF.

\subsection{Effect of PERM on anxiety-like behavior in the EPM}

ANOVA computed on the \% open arm entries $(F[1,12]=0.00973$, $P>0.05$ ) and on the \% time spent in the open arms $(F[1,12]=0.00973, P>0.05)$ revealed no significant group differences, reflecting the same level of anxiety-like responses in PERM treated rats and in controls.

\subsection{Effect of PERM on food consumption}

ANOVA revealed no difference between PERM treated rats and controls in food intake $(F[1,12]=0.750, P>0.05)$ neither a treatment $\times$ day interaction $(F[6 ; 72]=0.38, P>0.05)$ was observed.

\subsection{Effect of PERM on monoamines and their metabolites levels in Str, Hip and $m P F C$ areas}

As shown in Table 2, in the Str and in the Hip, a significant lower levels $(P<0.05)$ of DOPAC and DA were observed in PERM treated rats as compared to controls. Conversely, in the Str and Hip, the levels of HVA, a DA metabolite, were significantly higher $(P<0.05)$ in the PERM treated rats than in the controls. In addition,

Table 1

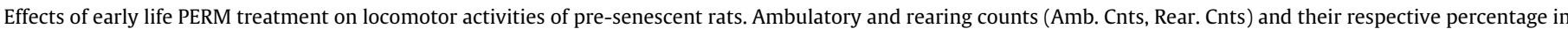
the center zone of the open field task (\% Amb. Cnts, \% Rear. Cnts) in 10 min. All data are expressed as mean \pm SEM. Group sizes: control $(N=7)$, PERM ( $N=7)$.

\begin{tabular}{|c|c|c|c|c|}
\hline Group & Amb. Cnts & Rear. Cnts & \% Amb. Cnts & \% Rear. Cnts \\
\hline Control & $2031.24 \pm 151.99$ & $94.57 \pm 4.65$ & $4.84 \pm 0.78$ & $1.20 \pm 0.46$ \\
\hline PERM & $1820.14 \pm 337.19$ & $96.57 \pm 19.11$ & $6.45 \pm 1.81$ & $1.04 \pm 0.73$ \\
\hline
\end{tabular}


Table 2

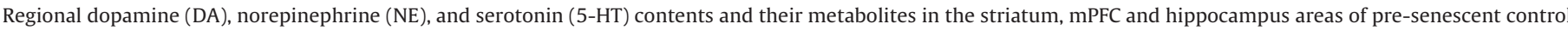

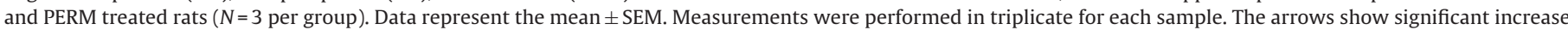
or decrease compared to the control group.

\begin{tabular}{|c|c|c|c|c|}
\hline Monoamines and metabolites & Treatment & Striatum (ng/mg tissue) & PFC (ng/mg tissue) & Hippocampus (ng/mg tissue) \\
\hline L-DOPA & $\begin{array}{l}\text { Control } \\
\text { PERM }\end{array}$ & $\begin{array}{l}0.173 \pm 7.0 \times 10^{-4} \\
0.103 \pm 4.0 \times 10^{-4^{*}} \downarrow\end{array}$ & $\begin{array}{l}0.029 \pm 5.0 \times 10^{-4} \\
0.033 \pm 4.8 \times 10^{-3}\end{array}$ & $\begin{array}{l}0.065 \pm 6.0 \times 10^{-4} \\
0.035 \pm 4.0 \times 10^{-4^{*}} \downarrow\end{array}$ \\
\hline DA & $\begin{array}{l}\text { Control } \\
\text { PERM }\end{array}$ & $\begin{array}{l}5.025 \pm 4.6 \times 10^{-3} \\
0.512 \pm 7.0 \times 10^{-4^{*}} \downarrow\end{array}$ & $\begin{array}{l}0.326 \pm 2.4 \times 10^{-3} \\
0.324 \pm 2.1 \times 10^{-3}\end{array}$ & $\begin{array}{l}0.181 \pm 3.0 \times 10^{-4} \\
0.094 \pm 6.0 \times 10^{-4^{*}} \downarrow\end{array}$ \\
\hline DOPAC & $\begin{array}{l}\text { Control } \\
\text { PERM }\end{array}$ & $\begin{array}{l}0.582 \pm 2.6 \times 10^{-3} \\
0.184 \pm 4.3 \times 10^{-3^{*}} \downarrow\end{array}$ & $\begin{array}{l}0.039 \pm 2.0 \times 10^{-4} \\
0.038 \pm 1.2 \times 10^{-3}\end{array}$ & $\begin{array}{l}0.035 \pm 4.0 \times 10^{-4} \\
0.036 \pm 1.7 \times 10^{-3}\end{array}$ \\
\hline HVA & $\begin{array}{l}\text { Control } \\
\text { PERM }\end{array}$ & $\begin{array}{l}0.062 \pm 8.0 \times 10^{-4} \\
0.079 \pm 4.0 \times 10^{-4^{*}} \uparrow\end{array}$ & $\begin{array}{l}0.006 \pm 1.0 \times 10^{-4} \\
0.006 \pm 1.0 \times 10^{-4}\end{array}$ & $\begin{array}{l}0.007 \pm 2.0 \times 10^{-4} \\
0.011 \pm 1.0 \times 10^{-4^{*}} \uparrow\end{array}$ \\
\hline $\mathrm{NE}$ & $\begin{array}{l}\text { Control } \\
\text { PERM }\end{array}$ & $\begin{array}{l}0.028 \pm 2.0 \times 10^{-4} \\
0.078 \pm 4.0 \times 10^{-4^{*}} \uparrow\end{array}$ & $\begin{array}{l}0.002 \pm 2.0 \times 10^{-4} \\
0.016 \pm 2.0 \times 10^{-4^{*}} \uparrow\end{array}$ & $\begin{array}{l}0.046 \pm 3.0 \times 10^{-4} \\
0.065 \pm 3.0 \times 10^{-4^{*}} \uparrow\end{array}$ \\
\hline MHPG & $\begin{array}{l}\text { Control } \\
\text { PERM }\end{array}$ & $\begin{array}{l}0.057 \pm 8.0 \times 10^{-4} \\
0.042 \pm 1.1 \times 10^{-3^{*}} \downarrow\end{array}$ & $\begin{array}{l}0.049 \pm 5.0 \times 10^{-4} \\
0.042 \pm 7.0 \times 10^{-4^{*}} \downarrow\end{array}$ & $\begin{array}{l}0.051 \pm 1.0 \times 10^{-3} \\
0.029 \pm 6.0 \times 10^{-4^{*}} \downarrow\end{array}$ \\
\hline 5-HT & $\begin{array}{l}\text { Control } \\
\text { PERM }\end{array}$ & $\begin{array}{l}1.152 \pm 8.4 \times 10^{-3} \\
1.182 \pm 7.4 \times 10^{-3}\end{array}$ & $\begin{array}{l}4.123 \pm 4.8 \times 10^{-3} \\
1.774 \pm 2.4 \times 10^{-2^{*}} \downarrow\end{array}$ & $\begin{array}{l}0.402 \pm 3.9 \times 10^{-3} \\
0.388 \pm 1.2 \times 10^{-2}\end{array}$ \\
\hline 5-HIAA & $\begin{array}{l}\text { Control } \\
\text { PERM }\end{array}$ & $\begin{array}{l}0.006 \pm 1.0 \times 10^{-4} \\
0.006 \pm 1.0 \times 10^{-4}\end{array}$ & $\begin{array}{l}0.016 \pm 1.0 \times 10^{-4} \\
0.023 \pm 7.0 \times 10^{-4^{*}} \uparrow\end{array}$ & $\begin{array}{l}0.006 \pm 1.0 \times 10^{-4} \\
0.006 \pm 2.0 \times 10^{-4}\end{array}$ \\
\hline Turnover ratios & Treatment & Striatum (ng/mg tissue) & PFC (ng/mg tissue) & Hippocampus (ng/mg tissue) \\
\hline DOPAC/DA & $\begin{array}{l}\text { Control } \\
\text { PERM }\end{array}$ & $\begin{array}{l}0.116 \pm 6.0 \times 10^{-4} \\
0.361 \pm 8.9 \times 10^{-3^{*}} \uparrow\end{array}$ & $\begin{array}{l}0.120 \pm 4.0 \times 10^{-4} \\
0.118 \pm 3.8 \times 10^{-3}\end{array}$ & $\begin{array}{l}0.198 \pm 2.2 \times 10^{-3} \\
0.388 \pm 1.5 \times 10^{-2^{*}} \uparrow\end{array}$ \\
\hline HVA/DA & $\begin{array}{l}\text { Control } \\
\text { PERM }\end{array}$ & $\begin{array}{l}0.012 \pm 1.0 \times 10^{-4} \\
0.154 \pm 9.0 \times 10^{-4^{*}} \uparrow\end{array}$ & $\begin{array}{l}0.019 \pm 2.0 \times 10^{-4} \\
0.020 \pm 5.0 \times 10^{-4}\end{array}$ & $\begin{array}{l}0.037 \pm 1.2 \times 10^{-3} \\
0.120 \pm 1.0 \times 10^{-3^{*}} \uparrow\end{array}$ \\
\hline MHPG/NE & $\begin{array}{l}\text { Control } \\
\text { PERM }\end{array}$ & $\begin{array}{l}2.065 \pm 4.6 \times 10^{-2} \\
0.540 \pm 1.4 \times 10^{-2^{*}} \downarrow\end{array}$ & $\begin{array}{l}28.44 \pm 3.5 \\
2.591 \pm 6.1 \times 10^{-2^{*}} \downarrow\end{array}$ & $\begin{array}{l}1.111 \pm 2.8 \times 10^{-2} \\
0.441 \pm 7.1 \times 10^{-3^{*}} \downarrow\end{array}$ \\
\hline 5-HIAA/5-HT & $\begin{array}{l}\text { Control } \\
\text { PERM }\end{array}$ & $\begin{array}{l}0.005 \pm 1.0 \times 10^{-4} \\
0.005 \pm 1.0 \times 10^{-4}\end{array}$ & $\begin{array}{l}0.004 \pm 2.7 \times 10^{-5} \\
0.013 \pm 4.0 \times 10^{-4^{*}}\end{array}$ & $\begin{array}{l}0.014 \pm 2.0 \times 10^{-4} \\
0.015 \pm 7.0 \times 10^{-4}\end{array}$ \\
\hline
\end{tabular}

${ }^{*} P<0.05$ vs. control.

the turnover ratios of DOPAC/DA and HVA/DA for the PERM treated group were much higher than that of the control group both in the $\operatorname{Str}(\mathrm{DOPAC} / \mathrm{DA}=3$ times; HVA/DA $=12$ times) and in the Hip (DOPAC/DA $=2$ times; HVA/DA $=3$ times).

A significant increase of NE levels in the Str, Hip and mPFC was observed in PERM treated rats as compared to controls $(P<0.05)$. Conversely, the levels of MHPG, a NE metabolite, were significantly $(P<0.05)$ reduced in Str, Hip and MPFC of PERM treated rats. The MHPG/NE turnover ratios of the PERM treated group, in the Str, Hip and $\mathrm{mPFC}$, were 4,11 and 2.5 times lower $(P<0.05)$ than that of the control group.

In the mPFC, a significant $(P<0.05)$ decrease of 5 -HT and a significant $(P<0.05)$ increase of 5-HIAA, its metabolite, were also observed. In the MPFC, the turnover ratio of 5-HIAA/5-HT in the PERM treated group was 3 times higher $(P<0.05)$ than in the controls.

Overall, these findings indicate that PERM treatment elicited a substantial reduction in DA and 5-HT levels and elevation in dopaminergic and serotonergic turnover. Conversely, following PERM treatment, NE levels were increased and its turnover decreased.

\subsection{Effect of PERM on glucose and lactate levels in the Str, Hip and $M P F C$}

As shown in Fig. 3, a significantly higher glucose $(P<0.05)$ and lower lactate $(P<0.05)$ concentrations were observed in the MPFC of PERM treated rats compared to controls. In particular, PERM treated rats had higher glucose $(P<0.05)$ and lower lactate $(P<0.05)$ levels compared with the control rats.

\section{Discussion}

Pyrethroid compounds are present at low concentrations in the environment and in human tissue. They are increasingly used as pesticides and their widespread presence is a cause of growing toxicological concerns. The pyrethroids are primarily metabolized in the liver of mammals by cytochrome P450 oxidation, or esterase hydrolysis followed by the formation of glucuronide and glycine conjugates. Common metabolites, such as the 3-PBA, are found in the urine of both laboratory animals and humans. However, there is a significant species differences in the metabolism of pyrethroids: carboxylesterases have been identified as the family of esterase which hydrolyze the pyrethroids only in the serum of rats. This would be of significant concern to human health as decreased metabolism of pyrethroids in humans could lead to increased expo-

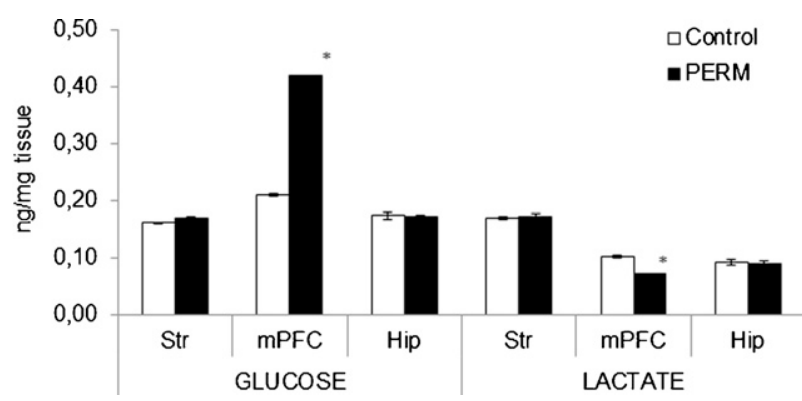

Fig. 3. Regional glucose and lactate levels measured in striatum, hippocampus and MPFC of pre-senescent control and PERM treated rats. Data represent the mean \pm SEM for three rats. Group sizes: $\operatorname{control}(N=3)$, PERM $(N=3)$. Measurements were performed in triplicate for each sample. ${ }^{*} P<0.05$ vs. control group. 
sure at the target tissue and increased potency (Godin et al., 2007). In addition, pyrethroids are highly lipophilic and by easily crossing the blood-brain-barrier they can reach the CNS at concentrations that can be potentially neurotoxic. Detrimental effects on CNS could be particularly pronounced if, as shown in our previous studies, exposure to these agents occurs in early life (from PND6 to PND21) when the rat brain is in a highly active neurodevelopmental phase as synaptogenesis. The choice to treat rats in the period derives from evidences reporting that exposure to pesticide in this period of life corresponds to the third trimester of pregnancy in human and it has been associated with the development of various diseases in adult and old age (Clancy et al., 2007; Vaiserman, 2011; Barker, 2001). For this reason we sought to determine the consequence of neonatal PERM exposure on late adulthood neurological functions.

Results revealed that PERM exposure from PND6 to PND21 did not elicited overt signs of toxicity in the rat. Animals showed a normal body weight gain curve and a normal feeding behavior. However, when during late adulthood PERM treated rats were tested for working memory performances, a memory form largely processed by the MPFC, in a T-maze-rewarded alternation task they took longer to choose for the correct arm in comparison to age matched controls. The PERM treated rats showed a clear trend to a higher number of consecutive incorrect arm choice. The difference from control was particularly evident during the first four days of training. A non-significant trend to difference in the percent of correct response was also observed.

In subsequent experiments neither differences in EPM anxietylike responses nor in OF locomotor activity were observed between PERM and control rats. Daily food intake and body weight were also identical in the two groups of animals. Altogether, these findings rule out the possibility that, in the T-maze, the different response between PERM and vehicle treated rats could depend upon differences in anxiety-like state, in locomotor activity or in feeding behavior. Most notably, however, it was found that in the water maze spatial orientation task, a type of memory that is mostly processed in the Hip, no differences were observed between PERM and vehicle treated rats.

In this regard, it should be argued that the spatial working memory necessary to complete a continuous foraging task (as our T-maze) is supported by integration of signals originated from multiple brain regions and encoding not only for allocentric (visuospatial signals existing in the external environment) representations of space, but also for egocentric (intrinsic representation based on the own movement patterns) stimuli (Oliveira et al., 1997; Gomez et al., 2009). Hippocampal activity is important for a configuration of an allocentric map (Olvera-Cortés et al., 2002, 2004; Poucet, 1993) that can then be translated into effective working memory performance in the PFC (Dégenètais et al., 2003] by virtue of the direct Hip-PFC connections (Cenquizca and Swanson, 2007). Whereas, for egocentric processing, connectivity between the Str and the MPFC appears to play a primary role (McDonald and White, 1994; Postle and D’Esposito, 2003). Hence, our findings showing a selective effect of PERM treatment on the T-maze task point to an involvement of frontal cortico-striatal circuitry rather than to a role for the Hip. To explore this hypothesis at neurochemical level, we measured the concentration of DA, NE, 5-HT and their metabolites in the PFC, Str and Hip. The most striking finding was in the Str where DA levels were decreased of about 10 times in PERM treated rats. A less pronounced reduction (two times) in DA concentration was also observed in the Hip. In these areas, a compensatory increase of DA turnover (DOPAC/DA and HVA/DA) was also observed. These results led us to speculate that DA depletion in Str and to some extent in the Hip could be responsible for the disruption of temporal perception (i.e., slowing the clock-speed) which could explain the reduction in the CRT following PERM treat- ment. In the T-maze task, our animals appeared more hesitant in choosing whether to go left or right to obtain the reward. This behavior has some similarities with that of patients with Parkinson's disease who have difficulty in initiating motor commands because of a lack of striatal DA resulting in an overactive indirect pathway leading to excessive cortical inhibition (Filion and Tremblay, 1991; Jellinger, 2002).

Contrary to DA, the NE level was increased in Hip (1.4 times), Str (2.8 times) and mPFC ( 8 times) of PERM treated rats compared to controls. As reported in previous studies, brain NE system activation may stimulate arousal and contribute to execution of goal oriented actions (Frazer, 2000; Aston-Jones et al., 1991). On the other hand, NE has an inverted U-shaped influence on these behavioral outcomes (Birnbaum et al., 2004). Hence, it is possible that if the NE system is excessively stimulated, as for example in the MPFC, it may mediate disruption of these behaviors. Finally, a significant reduction of 5-HT levels in the PFC of PERM treated rats was also observed. These data are in line with a classical view of a functional competition between NE and 5-HT in behavioral control (Ellison, 1979).

To further examine the possibility that these changes in catecholamine transmission might have resulted in a hypometabolic state of mPFC neurons, glucose and lactate concentrations in the mPFC were measured. To support this possibility, results revealed reduction in glucose consumption and lactate production hence indicating a significant impairment in energy metabolism in the mPFC of PERM treated animals as compared to controls.

It should be noted, however, that the amount of PERM given to animals led to higher urine concentration of its metabolite than those previously reported in children. In fact, at the end of the 15-day dosing period, the urinary 3-PBA concentration in rats was $41.50 \mu \mathrm{g} / \mathrm{L}(0.11 \pm 0.01 \mu \mathrm{g}$ in a mean urinary volume of $2.65 \mathrm{~mL})$, whereas in children the median (as 50th percentile) value ranged from $0.3 \mu \mathrm{g} / \mathrm{L}$ to $5.0 \mu \mathrm{g} / \mathrm{L}$ according to the different studies (Barr et al., 2010; Naeher et al., 2010; Becker et al., 2006; Zartarian et al., 2012). However, 3-PBA levels in children were also found as high as $20 \mu \mathrm{g} / \mathrm{L}$ at the 95 th percentile. Of note, the excretion curve of rats and humans (90\% of 3-PBA excreted within the first $24 \mathrm{~h}$ following exposure) remained identical (Kühn et al., 1999). Besides, in our rats the 3-PBA was completely eliminated $96 \mathrm{~h}$ after the last treatment.

Taken together, the present findings indicate that neonatal exposure (15 days) to $34 \mathrm{mg} / \mathrm{kg}$ of PERM could selectively alter cognitive functions associate with working memory processing. To our knowledge, at present it is unknown whether administration of lower doses of pyretroids for longer periods could induce cognitive dysfunctions similar to those observed in the present work. Further studies are warranted to explore this possibility.

\section{Conflict of interest statement}

The authors declare no conflict of interest.

\section{Acknowledgement}

This work was supported by a grant of the Italian Ministry of University and Research (no. 2008ZW3FJ3 to R.G.)

\section{References}

Aston-Jones, G., Chiang, C., Alexinsky, T., 1991. Discharge of noradrenergic locus coeruleus neurons in behaving rats and monkeys suggests a role in vigilance. Prog. Brain Res. 88, 501-520.

Barker, D.J.P., 2001. Fetal and infant origins of adult disease. Monatsschr. Kinderheilkd. 49, 2-6.

Barr, D.B., Olsson, A.O., Wong, L.Y., Udunka, S., Baker, S.E., Whitehead, R.D., Magsumbol, M.S., Williams, B.L., Needham, L.L., 2010. Urinary concentrations of 
metabolites of pyrethroid insecticides in the general U.S. population: National Health and Nutrition Examination Survey 1999-2002. Environ. Health Perspect. $118,742-748$.

Becker, K., Seiwert, M., Angerer, J., Kolossa-Gehring, M., Hoppe, H.W., Ball, M., 2006. GerES IV pilot study: assessment of the exposure of German children to organo phosphorus and pyrethroid pesticides. Int. J. Hyg. Environ. Health 209, 221-233.

Birnbaum, S.G., Yuan, P.X., Wang, M., Vijayraghavan, S., Bloom, A.K., Davis, D.J., Gobeske, K.T., Sweatt, J.D., Manji, H.K., Arnsten, A.F., 2004. Protein kinase C overactivity impairs prefrontal cortical regulation of working memory. Science 29 , 882-884.

Bleiberg, B., Steinberg, J.J., Katz, S.D., Wexler, J., LeJemtel, T., 1991. Determination of plasma lactic acid concentration and specific activity using high-performance liquid chromatography. J. Chromatogr. 23, 301-318.

Cannazza, G., Di Stefano, A., Mosciatti, B., Braghiroli, D., Baraldi, M., Pinnen, F., Sozio, P., Benatti, C., Parenti, C., 2005. Detection of levodopa, dopamine and its metabolites in rat striatum dialysates following peripheral administration of L-DOPA prodrugs by mean of HPLC-EC. J. Pharm. Biomed. Anal. 36, 1079-1084.

Cantalamessa, F., 1993. Acute toxicity of two parathyroid, permethrin, and cypermethrin in neonatal and adult rats. Arch. Toxicol. 67, 510-513.

Carloni, M., Nasuti, C., Fedeli, D., Montani, M., Amici, A., Vadhana, D.M.S., Gabbianelli, R., 2012. The impact of early life permethrin exposure on development of neurodegeneration in adulthood. Exp. Gerontol. 47, 60-66.

Cenquizca, L.A., Swanson, L.W., 2007. Spatial organization of direct hippocampal field CA1 axonal projections to the rest of the cerebral cortex. Brain Res. Rev. 56, $1-26$.

Clancy, B., Finlay, B.L., Darlington, R.B., Anand, K.J., 2007. Extrapolating brain development from experimental species to humans. Neurotoxicology 28, 931937.

Dégenètais, E., Thierry, A.M., Glowinski, J., Gioanni, Y., 2003. Synaptic influence of hippocampus on pyramidal cells of the rat prefrontal cortex: an in vivo intracellular recording study. Cereb. Cortex 13, 782-792.

Ellison, G., 1979. Chemical systems of the brain and evolution. In: Oakley, D., Plotkin, H. (Eds.), Brain, Behaviour and Evolution. Methuen, London, pp. 78-98.

Filion, M., Tremblay, L., 1991. Abnormal spontaneous activity of globus pallidus neurons in monkey with MPTP-induced parkinsonism. Brain Res. 547, 142-151.

Frazer, A., 2000. Norepinephrine involvement in antidepressant action. J. Clin. Psychiatry $61,25-30$.

Gallagher, M., Burwell, R., Burchinal, M., 1993. Severity of spatial learning impairment in aging: development of a learning index for performance in the Morris water maze. Behav. Neurosci. 107, 618-626.

Godin, S.J., Crow, J.A., Scollon, E.J., Hughes, M.F., DeVito, M.J., Ross, M.K., 2007. Identification of rat and human cytochrome $\mathrm{p} 450$ isoforms and a rat serum esterase that metabolize the pyrethroid insecticides deltamethrin and esfenvalerate. Drug Metab. Dispos. 35, 1664-1671.

Gomez-Villafuertes, R., Mellström, B., Naranjo, J.R., 2007. Searching for a role of NCX/NCKX exchangers in neurodegeneration. Mol. Neurobiol. 35, 195-202.

Gomez, A., Rousset, S., Baciu, M., 2009. Egocentric-updating during navigation facilitates episodic memory retrieval. Acta Psychol. Amst. 132, 221-227.

Heudorf, U., Angerer, J., 2001. Metabolites of pyrethroid insecticides in urine specimens: current exposure in an urban population in Germany. Environ. Health Perspect. 109, 213-217.

Imamura, L., Yasuda, M., Kuramitsu, K., Hara, D., Tabuchi, A., Tsuda, M., 2006. Deltamethrin, a pyrethroid insecticide, is a potent inducer for the activity dependent gene expression of brain-derived neurotrophic factor in neurons. J. Pharmacol. Exp. Ther. 316, 136-143.

Jellinger, K.A., 2002. Recent developments in the pathology of Parkinson's disease. J. Neural Transm. Suppl. 62, 347-376.

Kühn, K., Wieseler, B., Leng, G., Idel, H., 1999. Toxicokinetics of pyrethroids in humans: consequences for biological monitoring. Bull. Environ. Contam. Toxicol. $62,101-108$.
Leng, G., Kuhn, K.H., Idel, H., 1997. Biological monitoring of pyrethroid metabolites in urine of pest control operators. Toxicol. Lett. 88, 215-220.

McDonald, R.J., White, N.M., 1994. Parallel information processing in the water maze: evidence for independent memory systems involving dorsal striatum and hippocampus. Behav. Neural. Biol. 61, 260-270.

Naeher, L.P., Tulve, N.S., Egeghy, P.P., Barr, D.B., Adetona, O., Fortmann, R.C., Needham, L.L., Bozeman, E., Hilliard, A., Sheldon, L.S., 2010. Organophosphorus and pyrethroid insecticide urinary metabolite concentrations in young children living in a southeastern United States city. Sci. Total Environ. 408, 1145-1153.

Nakamura, Y., Sugihara, K., Sone, T., Isobe, M., Ohta, S., Kitamura, S., 2007. The in vitro metabolism of a pyrethroid insecticide, permethrin, and its hydrolysis products in rats. Toxicology 235, 176-184.

Narahashi, T., 1996. Neuronal ion channels as the target sites of insecticides. Pharmacol. Toxicol. 78, 1-14.

Nasuti, C., Gabbianelli, R., Falcioni, M.L., Di Stefano, A., Sozio, P., Cantalamessa, F., 2007. Dopaminergic system modulation, behavioural changes, and oxidative stress after neonatal administration of pyrethyroids. Toxicology 229, 194-205.

Nasuti, C., Falcioni, M.L., Nwankwo, I.E., Cantalamessa, F., Gabbianelli, R., 2008. Effect of permethrin plus antioxidants on locomotor activity and striatum in adolescent rats. Toxicology 251, 45-50.

Newman, L.A., Korol, D.L., Gold, P.E., 2011. Lactate produced by glycogenolysis in astrocytes regulates memory processing. PLoS ONE 6 http://dx.doi.org/10.1371/journal.pone.0028427.

Oliveira, M.G.M., Bueno, O.F.A., Pomarico, A.C., Gugliano, E.B., 1997. Strategies used by hippocampal- and caudate-putamen-lesioned rats in a learning task. Neurobiol. Learn. Mem. 68, 32-41.

Olvera-Cortés, E., Cervantes, M., González-Burgos, I., 2002. Place-learning, but not cue-learning training, modifies the hippocampal theta rhythm in rats. Brain Res. Bull. 58, 261-270.

Olvera-Cortés, E., Guevara, M.A., González-Burgos, I., 2004. Increase of the hippocampal theta activity in the Morris water maze reflects learning rather than motor activity. Brain Res. Bull. 62, 379-384.

Paxinos, G., Watson, C., 2007. The rat brain. In: Sterotaxic Coordinates, 6th ed. Academic Press, San Diego.

Pellerin, L., 2003. Lactate as a pivotal element in neuron-glia metabolic cooperation. Neurochem. Int. 43, 331-338.

Pellow, S., Chopin, P., File, S.E., Briley, M., 1985. Validation of open:closed arm entries in an elevated plus-maze as a measure of anxiety in the rat. J. Neurosci. Methods $14,149-167$.

Postle, B.R., D'Esposito, M., 2003. Spatial working memory activity of the caudate nucleus is sensitive to frame of reference. Cogn. Affect. Behav. Neurosci. 3 133-144.

Poucet, B., 1993. Spatial cognitive maps in animals: new hypotheses on their structure and neural mechanisms. Psychol. Rev. 100, 163-182.

Spijker, S., 2011. Dissection of rodent brain regions. In: Ka Wan Li (Ed.), Neuroproteomics. Springer, New York, pp. 13-26.

Tsacapoulos, M., Magistretti, P.J., 1996. Metabolic coupling between glia and neurons. J. Neurosci. 16, 877-885.

Uehara, T., Itoh, H., Matsuoka, T., Rujescu, D., Genius, J., Seo, T., Sumiyoshi, T., 2012 Effect of transient blockade of N-methyl-D-aspartate receptors at neonatal stage on stress-induced lactate metabolism in the medial prefrontal cortex of adult rats: role of 5-HT1A receptor agonism. Synapse 66, 408-417.

Vaiserman, A., 2011. Early-life origin of adult disease: evidence from natural experiments. Exp. Gerontol. 46, 189-192.

Wyss, M.T., Jolivet, R., Buck, A., Magistretti, P.J., Weber, B., 2011. In vivo evidence for lactate as a neuronal energy source. J. Neurosci. 31, 7477-7485.

Zartarian, V., Xue, J., Glen, G., Smith, L., Tulve, N., Tornero-Velez, R., 2012. Quantifying children's aggregate (dietary and residential) exposure and dose to permethrin: application and evaluation of EPA's probabilistic SHEDS-multimedia model. J. Expo. Sci. Environ. Epidemiol. 22, 267-273. 